\title{
The relationship between ambient temperature and FPG, a series of cross-sectional studies in Guangdong Province, China
}

Jiali Luo ${ }^{1,2 \#}$, Guanhao $\mathrm{He}^{2,4 \#}$, Yanjun $\mathrm{Xu}^{3}$, Zihui $\mathrm{Chen}^{2}$, Xiaojun $\mathrm{Xu}^{3}$, Shaowei Chen ${ }^{2}$, Jianxiong $\mathrm{Hu}^{2}$, Guiyuan $\mathrm{Ji}^{2}$, Tao Liu ${ }^{2}$, Weilin Zeng ${ }^{2}$, Xing $\mathrm{Li}^{2}$, Jianpeng $\mathrm{Xiao}^{2}$, Lingchuan $\mathrm{Guo}^{2}$, Wenjun $\mathrm{Ma}^{1,2^{*}}$

1. School of Public Health, Guangdong Pharmaceutical University, Guangzhou, 510310, China

2. Guangdong Provincial Institute of Public Health, Guangdong Provincial Center for Disease Control and Prevention, Guangzhou, 511430, China

3. Guangdong Provincial Center for Disease Control and Prevention, Guangzhou, 511430, China

4. School of Public Health, Southern Medical University, Guangzhou, 510515, China

\#: These authors contributed equally to this work

*: Correspondence to: Professor Wenjun Ma, No. 160, Qunxian Road, Panyu District, Guangzhou, Guangdong, 511430, China.

E-mail address: mawj@gdiph.org.cn (Professor Wenjun Ma)

Tel: +86-20-31051632, Fax: +86-20-31051652 (Professor Wenjun Ma) 


\section{Abstract}

2 Background: There existed evidence that the incidence and glycemic control rate of 3 T2DM have seasonal variation, which can be attributed to the effect of temperature on

4 FPG. The present study aimed to examine the associations between short-term ambient temperature exposure and FPG among different populations and calculate temperature-FPG association adjusted prevalence and glycemic control rate of T2DM.

7 Methods: Four cross-sectional health surveys with 26,350 respondents were conducted in Guangdong Province from 2007 to 2015. Multistage cluster sampling was used to recruit study participants. Gaussian generalized additive model was employed to evaluate the associations between daily mean ambient temperature and FPG among different populations (total, non-T2DM, old-T2DM and new-T2DM populations). Prevalence and glycemic control rate of T2DM were calculated based on the exposure-response association between temperature and FPG.

Results: The exposure-response curve of temperature and FPG were downward parabola in total, non-T2DM and old-T2DM populations, while the curve was "U"-shaped but not significant in new T2DM population. When temperature increased from $10^{\text {th }}$ percentile to $50^{\text {th }}$ percentile, the FPG significantly decreased 0.14 (95\%CI: $-0.17,-0.11) \mathrm{mmol} / \mathrm{L}, 0.11(95 \% \mathrm{CI}:-0.12,-0.11)$ and 0.44 (95\%CI: $-0.80,-0.08)$ in total, non-T2DM and old-T2DM populations, respectively. When temperature increased from $50^{\text {th }}$ to $90^{\text {th }}$ percentile, the FPG significantly decreased 0.19 (95\%CI: $-0.22,-0.16) \mathrm{mmol} / \mathrm{L}, 0.17$ (95\%CI: $-0.17,-0.17)$ and 0.51 (95\%CI: $-0.79,-0.23)$ in total, non-T2DM and old-T2DM populations, respectively. The alteration of rate related to the temperature difference was observed from $5^{\circ} \mathrm{C}$ to $30^{\circ} \mathrm{C}$, with gently decreases from $10.03 \%$ to $9.39 \%$ in prevalence of T2DM and greatly increases from $32.1 \%$ to $58.6 \%$ in glycemic control rate of T2DM, respectively.

Conclusion: FPG, the prevalence and glycemic control rate of T2DM are affected by ambient temperature, which suggests temperature and FPG association should be 
29 clinical management of T2DM.

30 Keywords Ambient temperature, Fasting plasma glucose, T2DM, prevalence, 31 glycemic control rate. 


\section{Background}

Type 2 diabetes mellitus (T2DM) is a metabolic disorder characterized by high blood glucose in the context of insulin resistance and relative insulin deficiency. T2DM has been a very serious public health problem worldwide and accounted for about $90 \%$ of diabetes cases. It was estimated that diabetes affected more than 425 million people worldwide in 2017, and 114.4 million diabetes cases were in China [1]. Diabetes is a major contributor to cardiovascular diseases and is the eleventh common cause of disability worldwide [1].

Many risk factors such as heredity, individual life style and insufficient activity are associated with T2DM [2-4]. Environmental factors such as seasonal variation and ambient temperature were related to fasting plasma glucose (FPG). There was much evidence on seasonal variation of FPG. For example, there was a mean $0.6 \mathrm{mmol} / \mathrm{L}$ difference in FPG between winter and spring in Southern California [5]. Gikas et al. found that mean FPG was higher during cold seasons than warm months in a diabetic clinic study, with the nadir in August $(7.60 \mathrm{mmol} / \mathrm{L})$ and the zenith in February $(9.12$ $\mathrm{mmol} / \mathrm{L}$ ) [6]. In terms of acute effect of ambient temperature, previous studies have observed a negative relationship between temperature and FPG [5, 7]. Nevertheless, $L i$ et al. found that the association between temperature and FPG was U-shaped that FPG level was higher in cold or hot temperature [8].

There were also exited evidence that the incidence of T2DM and glycemic control rate had seasonal variation. A population-based study in Csongrad, Hungary revealed the seasonal variation in the incidence of T2DM that the peak occurred on March and the trough occurred on August [9]. Several studies also revealed seasonal variation of glycemic control rate for T2DM cases that lower control rate emerged in cold winter $[6,10]$. These seasonal variations can be attributed to temperature variation to a certain extent.

Despite the confirmed effects of ambient temperature on FPG as well as the prevalence and glycemic control rate of T2DM, previous epidemiological surveys 
60 conducted in different regions or seasons did not adjust temperature-FPG association 61 when computing T2DM prevalence and control rate [11]. However, different 62 nutritional prescription and hypoglycemic drug therapy made for diabetic cases by 63 considering temperature-FPG association can help achieve better diabetic clinic care 64 and management.

65 In order to fill the knowledge gap, in the current study, we examined the associations 66 of ambient temperature with FPG in different populations based on a series of 67 cross-sectional surveys, and further calculated temperature-FPG association adjusted 68 prevalence and control rate of T2DM. Our findings are informative for accurate 69 estimate prevalence of T2DM in large-scale surveys across different climate zones 70 and seasons, and clinic management of cases with T2DM in different seasons. 


\section{Methods}

\section{Study design and population}

The Guangdong Chronic Disease and Risk Factors Surveys are a series of provincially representative investigation, which conducted by Guangdong Provincial Center for Disease Control and Prevention in 2007, 2010 and 2013. These surveys aimed to monitor the prevalence trend and risk factors of non-communicable diseases such as hypertension, T2DM, and obesity. The Guangdong Nutrition and Health Survey conducted in 2015 was designed to assess the nutrition and health status in Guangdong province. The data from the four surveys were combined to examine the association of ambient temperature with FPG. All participants agreed to participate and signed informed consents form prior to the surveys. The study was approved by the Ethics Committee of Guangdong Provincial Center for Disease Control and Prevention.

Similar sampling methods and protocols were adopted for the surveys of 2007, 2010 and 2013, which has been described previously elsewhere [12]. Briefly, in each of the surveys, 21 districts or counties in Guangdong province were randomly selected by stratified multistage cluster sampling with probability proportional to size. In the second stage, four neighborhoods or townships from each district or county were selected; In the third stage, three communities or villages from each neighborhood or township were selected; In the fourth stage, 50 to 100 households from each community or village were randomly selected; Finally, 1 resident aged $\geq 18$ years from each sampled household was selected using the Kish grid method. If there is no resident aged $\geq 18$ years in the selected household or if the selected resident did not agree to participate in the survey, the household was replaced with another randomly selected household nearby. Details of sampling methods and survey protocols for the nutrition and health survey conducted in 2015 have been described in the previous study [13]. 
Participants were asked to fast at least 8 hours before blood collection. Fasting blood samples were collected by registered nurses. Fasting plasma glucose levels were measured on a Hitachi 7600 automatic biochemical analyzer (Hitachi, Ltd., Tokyo, Japan) using reagents obtained from Wako Pure Chemical Industries Ltd. at the National CDC of China. Old-T2DM population was defined as physician-diagnosed T2DM (confirmed with medical history). According to World Health Organization 2006 criteria, new-T2DM population was defined a new discovery of diabetes with an FPG level of $7.0 \mathrm{mmol} / \mathrm{L}$ or greater among undiagnosed diabetes; non-T2DM population was defined as participants with an FPG level less than $7.0 \mathrm{mmol} / \mathrm{L}$ [14]. T2DM prevalence was as the proportion of old-T2DM patients and undiagnosed diabetes with FPG level less than $7.0 \mathrm{mmol} / \mathrm{L}$. Control was defined as the proportion of old-T2DM population with FPG less than $7.0 \mathrm{mmol} / \mathrm{L}$ [14].

\section{Data collection}

\section{Questionnaire and anthropometric Measurements}

Participants were interviewed and provided with onsite health examinations. All interviews and examinations were conducted following standard protocols by physicians who had received specific training for the survey and health examination. Questionnaires were used to collect a wide range of information including demographic characteristics, lifestyle, household location. Demographic characteristics included age, gender (male vs female), career (production staff vs technical staff vs nonworks/ houseworkers/retirees), education (less than junior high school vs high school/college). Physical activity time was defined as leisure time spend in high intensity sports or moderate intensity exercise, such as running, swimming, doing Tai Chi (in hour/day). Sedentary leisure time was defined as time spent in sitting-down activities outside work, such as watching TV, reading a newspaper and using a computer (in hour/day). Smoking status was measured by whether individual smoked in the past or present (yes vs no). Drinking status was defined as whether individual drank alcohol in the past 12 months (yes vs no). Height 
and weight were measured following standard protocols. Body mass index (BMI) was calculated as weight divided by the square of standing height (in $\mathrm{kg} / \mathrm{m}^{2}$ ).

\section{Meteorological data}

Daily meteorological data were obtained from the China National Weather Data Sharing System (http://data.cma.cn/). Monitoring locations included thirty-six areas and the number of survey areas was shown in Additional file 1: table S1. Daily meteorological variables from each survey's nearby weather station included daily mean, minimum, maximum temperature $\left({ }^{\circ} \mathrm{C}\right)$, relative humidity $(\%)$ and sunlight (hour/day), which will be matched to other individual survey information based on the date of survey. Daily meteorological data was passed through quality control checks. The integrity of ach meteorological data was closely to $99.9 \%$.

\section{Statistical analysis}

We characterized distributions of all variables, continuous variables were expressed as the means \pm SD for normally distributed data and mean (IQR, 25\%-75\%) for skew distributed data. Categorical variables were expressed as numbers and percentages. T-test (for normally distributed continuous data), Kruskal-Wallis test (for skew distributed continuous data) or $\chi^{2}$ test (for categorical variables) were used to compared the characteristics between non-T2DM, old T2DM and new T2DM participants. A generalized additive Gaussian models was used to investigate ambient temperature exposure on FPG among different populations after adjusting for covariates. Daily mean temperature was selected as exposure variable from daily minimum/mean/maxi-mum temperature according to the minimum value of Akaike' $\mathrm{s}$ Information criterion $(A I C)$ in the model (Additional file 2: figure S1). And daily mean temperature, humidity, age and BMI were fitted using a penalized cubic spline function with a degree of freedom (df) of 3 . Selection optimal $d f$ of daily mean temperature was based on graphic smoothness and minimum value of AIC (Additional file 3: figure S2). The regression model was established as following $Y_{i m}(1)$. 


$$
\begin{aligned}
Y_{\text {im }}= & \beta_{0 m}+\beta_{1 m} s\left(X_{\text {temp }}, k=3\right)+\beta_{2 m} s\left(X_{\text {humidity }}, k=3\right)+\beta_{3 m} s\left(X_{\text {age }}, k=3\right)+\beta_{4 m} s\left(X_{B M I}, k=3\right) \\
& +\beta_{5 m} X_{1 i}+\cdots+\beta_{n m} X_{n i}+\epsilon_{\text {im }}
\end{aligned}
$$

Where $m$ represents to groups (total/non-T2DM/new-T2DM/old-T2DM participants), $Y_{i m}$ represents to participant's FPG; $\beta_{0 m}$ is the overall intercept, $\beta_{1 m} \ldots \beta_{n m}$ corresponds to coefficients for variables. $X_{\text {temp }}, X_{\text {humidity }}, X_{\text {age }}, X_{B M I}$ and $X_{1 i} \ldots X_{n i}$ denotes variables. $S()$ is a penalized cubic spline function and $\epsilon_{i m}$ is the residual error.

After constructing the model, we obtained the exposure-response curve between daily mean temperature and the change of FPG compared to $50^{\text {th }}$ percentiles of temperature. We used the change of FPG at different typical interval $\left(10^{\text {th }}, 50^{\text {th }}, 90^{\text {th }}\right.$ percentiles of temperature) of the curve to demonstrate the effect of temperature. Based on exposure-response curve, we can obtain participant's change of FPG $\left(\triangle F P G_{i j m}\right)$ at survey day of temperature compared to $5^{\circ} \mathrm{C}, 10^{\circ} \mathrm{C}, 15^{\circ} \mathrm{C}, 20^{\circ} \mathrm{C}, 22.5^{\circ} \mathrm{C}, 25^{\circ} \mathrm{C}$ and $30^{\circ} \mathrm{C}$. Temperature-adjusted FPG $\left(\mathrm{FPG}_{2 \mathrm{ijm}}\right)(2)$, prevalence $\left(\right.$ Rate $\left._{1 \mathrm{j}}\right)(3)$ and glycemic control rate $\left(\right.$ Rate $\left._{2 \mathrm{j}}\right)(4)$ of T2DM can be calculated as the follows:

$$
F P G_{2 i j m}=F P G_{1 i m}-\triangle F P G_{i j m}
$$

$$
\text { Rate }_{1 j}=N_{l j} / N_{3}
$$

$$
\text { Rate }_{2 j}=N_{2 j} / N_{4}
$$

Where $m$ corresponds to groups (total/non-T2DM/new-T2DM/old-T2DM participants), $j$ represents temperature reference points $\left(5^{\circ} \mathrm{C}, 10^{\circ} \mathrm{C}, 15^{\circ} \mathrm{C}, 20^{\circ} \mathrm{C}\right.$, $22.5^{\circ} \mathrm{C}, 25^{\circ} \mathrm{C}$ and $\left.30^{\circ} \mathrm{C}\right) . \triangle F P G_{i j m}$ corresponds to participant's change of FPG at survey day of temperature compared to temperature reference points. $F P G_{\text {lim }}$ corresponds to each participant's FPG; $N_{l j}$ represents the sum of the number of old \& new T2DM patients and the number of non-T2DM population with $F P G_{2 i j m}$ level of 7 $\mathrm{mmol} / \mathrm{L}$ or greater; $N_{3}$ is number of total population; $N_{2 j}$ represents the number of old-T2DM with a $F P G_{2 i j m}$ less than $7 \mathrm{mmol} / \mathrm{L} ; N_{4}$ is the number of old-T2DM patients.

For sensitivity analysis, we analysis the association between ambient temperature and 
181 FPG across different lags (lag1 to lag6). We further added sunshine and precipitation 182 to the model to test the robustness of that association. We used R software (version 183 3.5.1, R foundation for Statistical Computing, Vienna, Austria). All statistical tests 184 were two-sided and $P$ values of all statistical analyses less than 0.05 was considered 185 statistically significant. The GAM analysis was performed by using package " $m g c v$ ". 


\section{Characteristics of study participants}

188

189

190

191

192

193

194 195

196

197

198

199

200

201

202

203

204

205

206

207

208

At baseline, total sample size was 26350 , and $90.6 \%$ of subjects were non-T2DM; $5.8 \%$ of subjects were new-T2DM and 3.5\% were old-T2DM. Overall, the mean (IQR) age of respondents was 50.1 (40.0-60.1) years old and $44.9 \%$ of them were males. Compared with non-T2DM participants, old-T2DM and new-T2DM participants were older and had higher levels in BMI, but lower education level. Moreover, there were more nonworkers/houseworkers/retirees, non-smokers and non-drinkers in old-T2DM and new-T2DM participants than non-T2DM participants (table 1). Density distribution of FPG among different populations and statistical description of FPG and daily mean temperature showed in additional file 4: figure $\mathrm{S} 3$ and additional file 5: table S2.

\section{Monthly variation in FPG and ambient temperature}

Fig 1 and additional file 6: Table S3 show monthly variation in FPG and ambient temperature. The climate is characterized by two main seasons: cold season (lasting from December to March) and warm season (April, May, October, November). Mean FPG levels were significantly higher during the cold season than warm season. The zenith of FPG was $6.57 \mathrm{mmol} / \mathrm{L}$ for total participants, $5.55 \mathrm{mmol} / \mathrm{L}$ for non-T2DM participants, $9.41 \mathrm{mmol} / \mathrm{L}$ for old T2DM participants, and $10.04 \mathrm{mmol} / \mathrm{L}$ for new T2DM participants in January or February or March, while the nadir of FPG was 5.29 $\mathrm{mmol} / \mathrm{L}$ for total participants, $5.05 \mathrm{mmol} / \mathrm{L}$ for non-T2DM participants, $7.39 \mathrm{mmol} / \mathrm{L}$ for old T2DM participants and $8.99 \mathrm{mmol} / \mathrm{L}$ for new T2DM participants in October or November. 
Table 1. Characteristic of study participants among different populations.

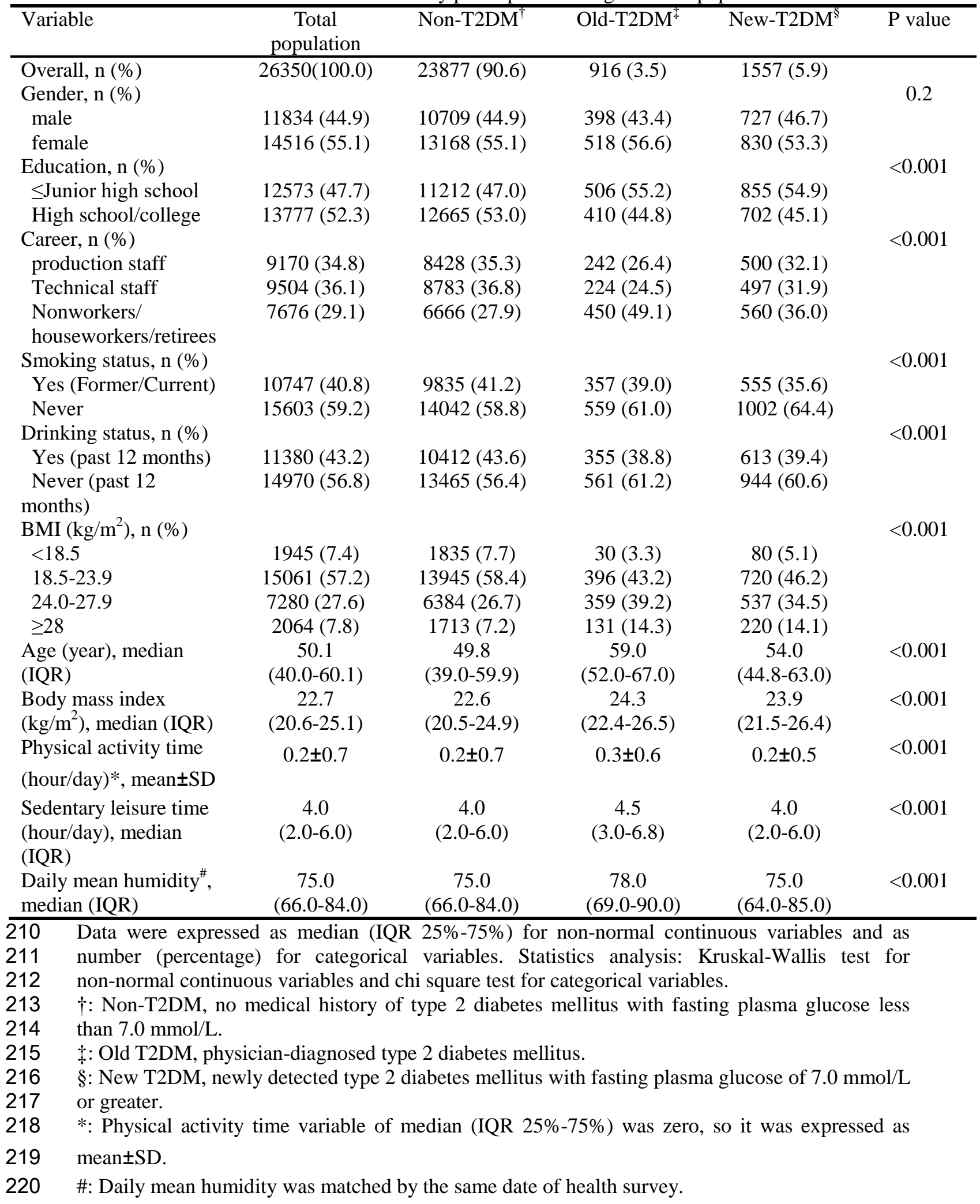



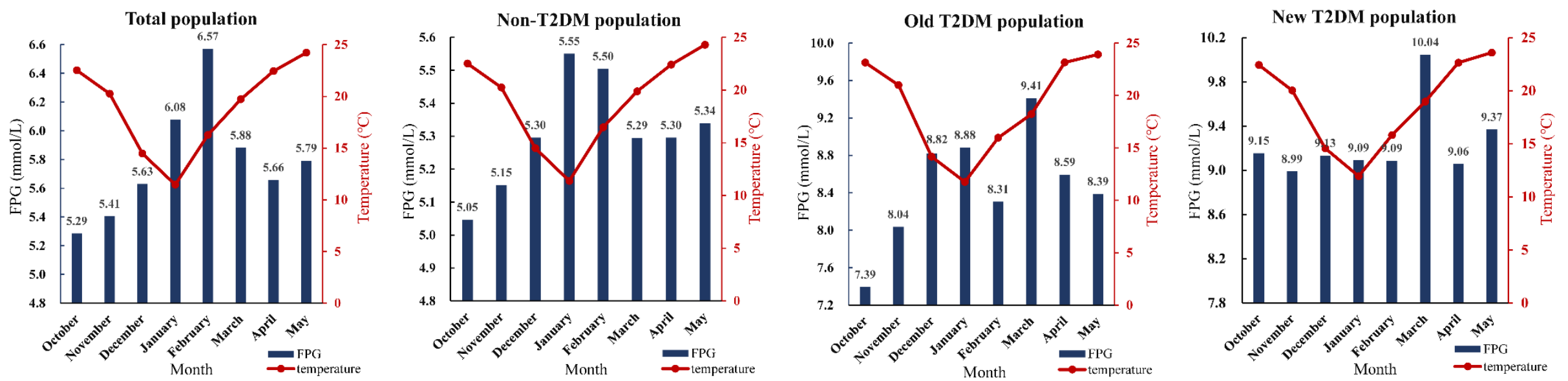

Figure1. Monthly variation in mean of FPG $(\mathrm{mmol} / \mathrm{L})$ and ambient temperature $\left({ }^{\circ} \mathrm{C}\right)$ among total, non-T2DM, old T2DM and new T2DM populations. 
224 In total, non-T2DM, old-T2DM populations, the exposure-response curves were 225 general downward parabola. As temperature increased FPG concentration 226 significantly decreased and the rate of descent saw an increasing trend. However, the 227 exposure-response curve was "U"-shaped but the effect of temperature was not 228 statistically significant (Figure 2).

229 In the first day of temperature exposure (lag0), when temperature increased from $10^{\text {th }}$ 230 percentile to $50^{\text {th }}$ percentile, the FPG significantly decreased 0.14 (95\% CI: -0.17 , $231-0.11) \mathrm{mmol} / \mathrm{L}, 0.11$ (95\%CI: -0.12, -0.11) and 0.44 (95\%CI: -0.80, -0.08) in total, 232 non-T2DM and old-T2DM populations, respectively, while we found no significant 233 effect of temperature in new-T2DM participants $(-0.19,95 \% \mathrm{CI}:-0.50,0.12)$. In lag0, 234 when temperature increased from $50^{\text {th }}$ to $90^{\text {th }}$ percentile, the FPG significantly 235 decreased 0.19 (95\%CI: -0.22, -0.16) mmol/L, 0.17 (95\%CI: $-0.17,-0.17)$ and 0.51 236 (95\% CI: $-0.79,-0.23$ ) in total, non-T2DM and old-T2DM populations, respectively, 237 while we found no significant effect of temperature in new-T2DM participants $(0.10$, 238 95\%CI: -0.19, 0.39) (Table 2 and figure 3).

\section{Temperature-adjusted FPG, prevalence and glycemic control rate of T2DM}

240 Temperature adjusted FPG decreased when temperature increased for total, 241 non-T2DM and old T2DM populations. For instance, the mean of 242 temperature-adjusted $\mathrm{FPG}$ was $5.78 \mathrm{mmol} / \mathrm{L}$ at $5^{\circ} \mathrm{C}$ while $5.22 \mathrm{mmol} / \mathrm{L}$ at $30^{\circ} \mathrm{C}$ in 243 total participants; $5.40 \mathrm{mmol} / \mathrm{L}$ at $5^{\circ} \mathrm{C}$ while $4.93 \mathrm{mmol} / \mathrm{L}$ at $30^{\circ} \mathrm{C}$ in non-T2DM 244 participants, $9.04 \mathrm{mmol} / \mathrm{L}$ at $5^{\circ} \mathrm{C}$ while $7.49 \mathrm{mmol} / \mathrm{L}$ at $30^{\circ} \mathrm{C}$ in old $\mathrm{T} 2 \mathrm{DM}$ 245 participants. However, the association between temperature and temperature-adjusted 246 FPG was a down and up trend in new T2DM population, such as the mean of temperature-adjusted $\mathrm{FPG}$ was $9.56 \mathrm{mmol} / \mathrm{L}$ at $5^{\circ} \mathrm{C}, 9.08 \mathrm{mmol} / \mathrm{L}$ at $20^{\circ} \mathrm{C}$ and 9.36

$248 \mathrm{mmol} / \mathrm{L}$ at $30^{\circ} \mathrm{C}$, respectively (figure $4 \mathrm{~A}$ ).

249 Temperature-FPG association adjusted prevalence and glycemic control rate of T2DM 
250 showed in figure4B. Based on temperature adjusted FPG, low ambient temperature 251 resulted in gently increased of prevalence of T2DM and greatly decreased of glycemic 252 control rate of T2DM. The alteration of rate related to the temperature difference was 253 observed from $5^{\circ} \mathrm{C}$ to $30^{\circ} \mathrm{C}$, with gently decreases from $10.03 \%$ to $9.39 \%$ in 254 prevalence of T2DM and greatly increases from $32.1 \%$ to $58.6 \%$ in glycemic control 255 rate of T2DM, respectively.

\section{Sensitivity analyses}

257 Similar exposure-response relationships between temperature and FPG were obtained 258 using different lag times or additionally adjusted sunshine and precipitation, which 259 suggested a robust relationship (Additional file 7: Figure S4 and Additional file 8: 260 Figure S5). And there were no significant change effects at different typical interval of 261 temperature on FPG compared to the result of the main model analysis, suggesting 262 that our results were relatively robust (Table 2 and figure 3 ). 
Total

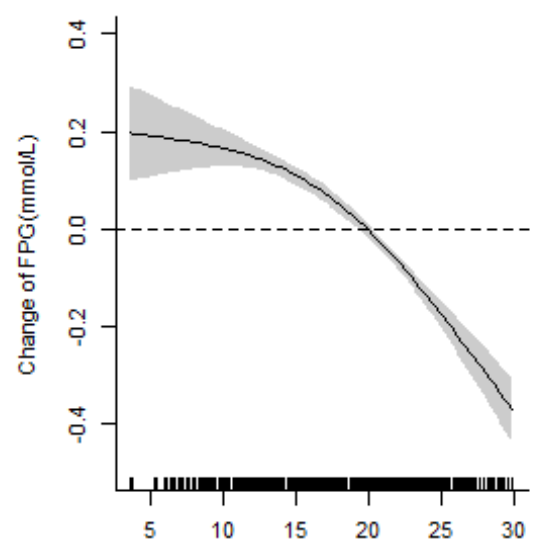

Daily mean temperature $(\mathrm{C})$
Non-T2DM

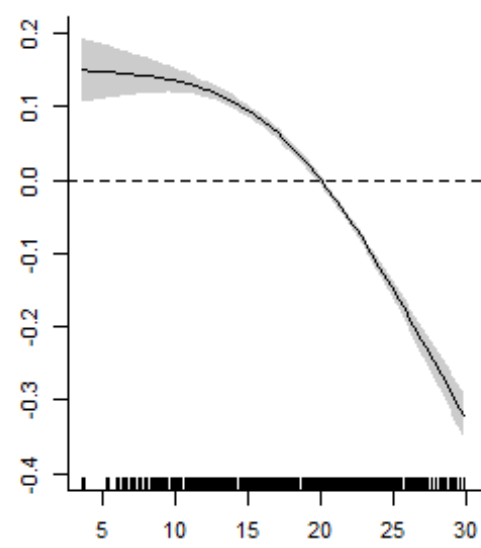

Daily mean temperature $(\mathrm{C})$
Old-T2DM

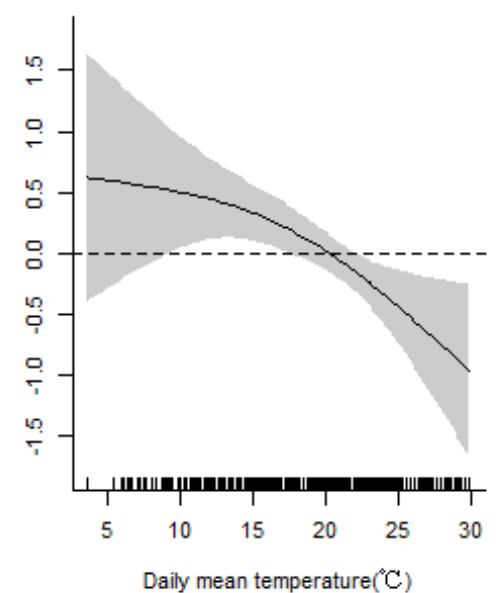

New-T2DM

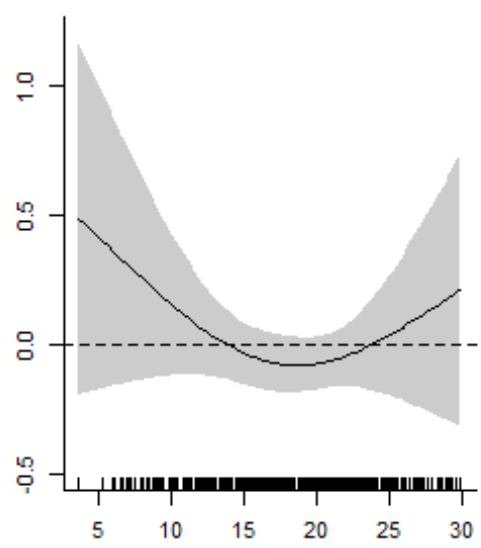

Daily mean temperature("C)

Figure 2. The exposure-response of daily mean temperature and FPG among total, non-T2DM, old T2DM and new T2DM populations.

Shade represent 95\%CI Confidence band. Non-T2DM, no medical history of type 2 diabetes mellitus with fasting plasma glucose less than 7.0 mmol/L. Old T2DM, physician-diagnosed type 2 diabetes mellitus. New T2DM, newly detected type 2 diabetes mellitus with fasting plasma glucose of $7.0 \mathrm{mmol} / \mathrm{L}$ or greater. Gaussian variables. 
Table 2 Change of FPG (mmol/L) at different typical interval of temperature.

\begin{tabular}{|c|c|c|c|c|c|c|c|c|}
\hline & \multicolumn{4}{|c|}{ Interval 1 of temperature $\left(\mathrm{P} 10(\mathrm{ref})\right.$ vs $\left.\mathrm{P} 50^{\#}\right) *$} & \multicolumn{4}{|c|}{ Interval 2 of temperature $\left(\mathrm{P} 50^{\#}\right.$ (ref) vs $\left.\mathrm{P} 90\right) *$} \\
\hline & Total & Non-T2DM ${ }^{\dagger}$ & Old T2DM $^{*}$ & New T2DM ${ }^{\S}$ & Total & Non-T2DM ${ }^{\dagger}$ & Old T2DM & New T2DM ${ }^{\S}$ \\
\hline Temperature & $11.3^{\circ} \mathrm{C}$ vs $19.4^{\circ} \mathrm{C}$ & $11.4^{\circ} \mathrm{C}$ vs $19.4^{\circ} \mathrm{C}$ & $10.9^{\circ} \mathrm{C}$ vs $19.8^{\circ} \mathrm{C}$ & $11.0^{\circ} \mathrm{C}$ vs $18.5^{\circ} \mathrm{C}$ & $19.4^{\circ} \mathrm{C}$ vs $25.1^{\circ} \mathrm{C}$ & $19.4^{\circ} \mathrm{C}$ vs $25.1^{\circ} \mathrm{C}$ & $19.8^{\circ} \mathrm{C}$ vs $25.3^{\circ} \mathrm{C}$ & $18.5^{\circ} \mathrm{C}$ vs $24.6^{\circ} \mathrm{C}$ \\
\hline \multicolumn{9}{|l|}{ Main model } \\
\hline Lag 0 & $-0.14(-0.17,-0.11)$ & $-0.11(-0.12,-0.11)$ & $-0.44(-0.80,-0.08)$ & $-0.19(-0.50,0.12)$ & $-0.19(-0.22,-0.16)$ & $-0.17(-0.17,-0.17)$ & $-0.51(-0.79,-0.23)$ & $0.10(-0.19,0.39)$ \\
\hline Lag 1 & $-0.14(-0.17,-0.12)$ & $-0.11(-0.11,-0.11)$ & $-0.42(-0.83,-0.01)$ & $-0.21(-0.52,0.10)$ & $-0.17(-0.20,-0.15)$ & $-0.16(-0.16,-0.16)$ & $-0.26(-0.58,0.06)$ & $0.12(-0.19,0.42)$ \\
\hline Lag 2 & $-0.16(-0.19,-0.13)$ & $-0.11(-0.12,-0.11)$ & $-0.52(-0.93,-0.11)$ & $-0.18(-0.45,0.09)$ & $-0.19(-0.21,-0.16)$ & $-0.16(-0.16,-0.16)$ & $-0.34(-0.67,-0.02)$ & $0.00(-0.26,0.27)$ \\
\hline Lag 3 & $-0.15(-0.18,-0.12)$ & $-0.12(-0.13,-0.12)$ & $-0.37(-0.68,-0.06)$ & $-0.11(-0.38,0.15)$ & $-0.22(-0.25,-0.19)$ & $-0.17(-0.17,-0.16)$ & $-0.61(-0.83,-0.40)$ & $0.03(-0.22,0.28)$ \\
\hline $\operatorname{Lag} 4$ & $-0.11(-0.14,-0.08)$ & $-0.07(-0.09,-0.05)$ & $-0.48(-0.83,-0.12)$ & $-0.13(-0.33,0.07)$ & $-0.24(-0.27,-0.22)$ & $-0.16(-0.19,-0.14)$ & $-0.61(-0.84,-0.39)$ & $-0.12(-0.30,0.07)$ \\
\hline Lag 5 & $-0.11(-0.14,-0.07)$ & $-0.06(-0.08,-0.03)$ & $-0.32(0.55,-0.09)$ & $-0.16(-0.38,0.06)$ & $-0.24(-0.27,-0.22)$ & $-0.18(-0.21,-0.15)$ & $-0.84(-0.96,-0.71)$ & $-0.13(-0.31,0.05)$ \\
\hline Lag 6 & $-0.08(-0.12,-0.05)$ & $-0.04(-0.08,-0.01)$ & $-0.31(-0.55,-0.07)$ & $-0.01(-0.24,0.21)$ & $-0.24(-0.27,-0.22)$ & $-0.20(-0.23,-0.16)$ & $-0.76(-0.93,-0.59)$ & $-0.01(-0.19,0.17)$ \\
\hline Model 2 & $-0.13(-0.16,-0.10)$ & $-0.11(-0.11,-0.10)$ & $-0.40(-0.76,-0.04)$ & $-0.16(-0.48,0.15)$ & $-0.16(-0.19,-0.13)$ & $-0.15(-0.15,-0.15)$ & $-0.45(-0.73,-0.16)$ & $0.12(-0.18,0.42)$ \\
\hline
\end{tabular}

$270 \uparrow:$ Non-T2DM, no medical history of type 2 diabetes mellitus with fasting plasma glucose $<7.0 \mathrm{mmol} / \mathrm{L}$.

271 t: Old T2DM, physician-diagnosed type 2 diabetes mellitus.

272 §: New T2DM, newly detected type 2 diabetes mellitus with fasting plasma glucose $\geq 7.0 \mathrm{mmol} / \mathrm{L}$.

273 \#: $50^{\text {th }}$ percentiles (P50) of daily mean temperature: total and non-T2DM were at $19.4^{\circ} \mathrm{C}$, old-T2DM was at $19.8^{\circ} \mathrm{C}$ and new-T2DM was at $18.5^{\circ} \mathrm{C}$.

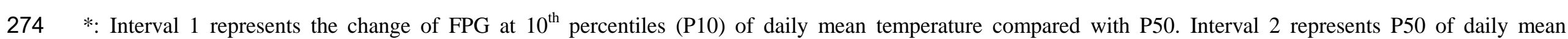

275 temperature compared with $90^{\text {th }}$ percentiles (P90).

276 Main model was adjusted for age, sex, BMI, education, career, physical activity, sedentary leisure times, smoking status, drinking status and humidity variables.

277 Model2 were adjusted model 1 and additionally sunshine and precipitation variables. 

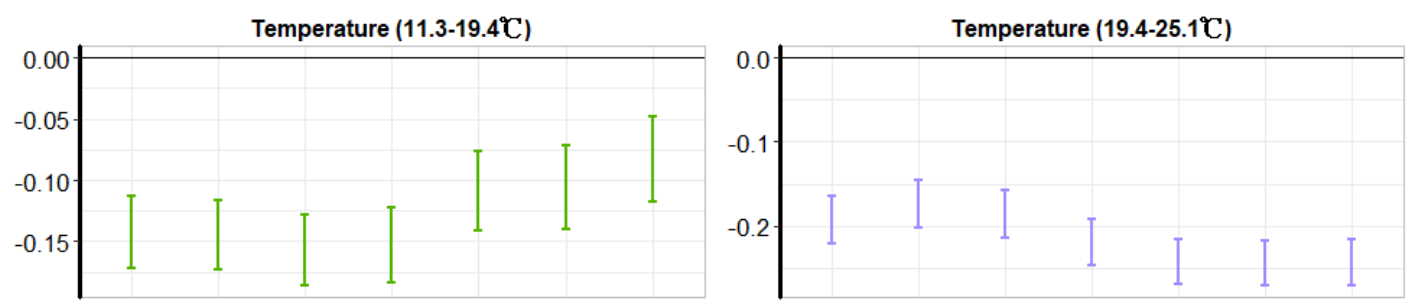

Non-T2DM

Non-T2DM
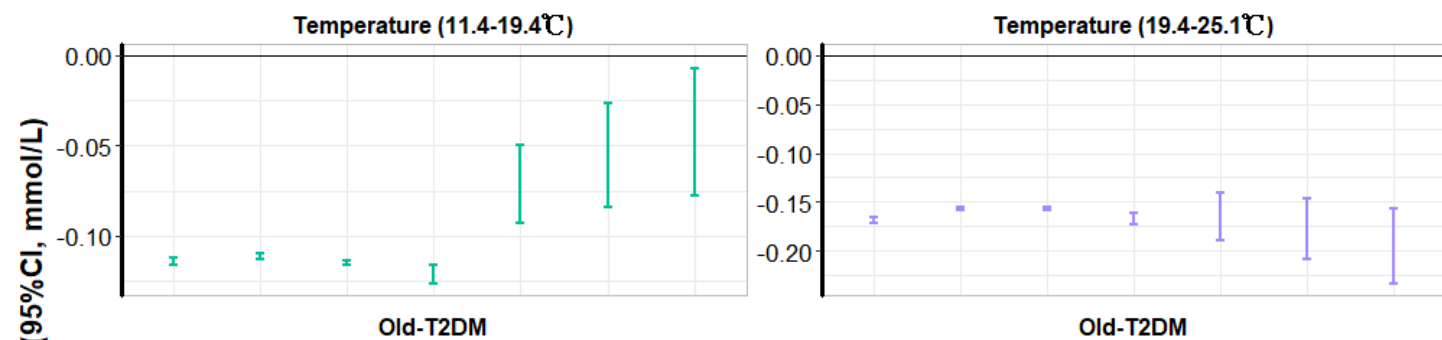

Old-T2DM
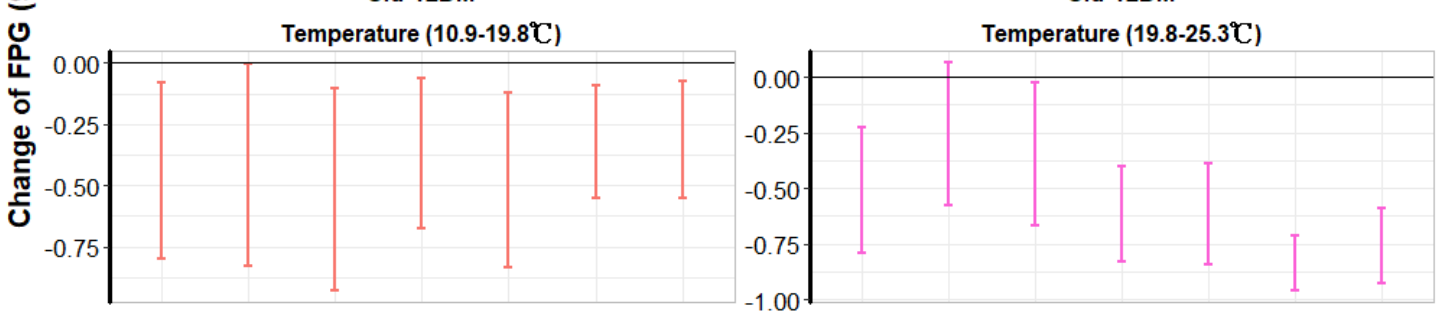

New-T2DM

New-T2DM
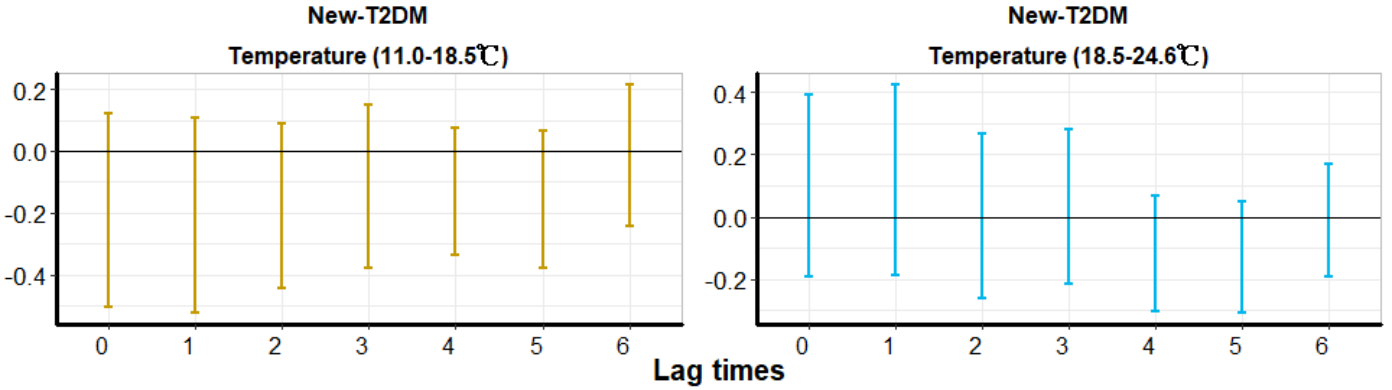

279 Figure 3. The effect of temperature on FPG with different lag times. $50^{\text {th }}$ percentiles (P50) of daily 280 mean temperature: total and non-T2DM were at $19.4^{\circ} \mathrm{C}$, old-T2DM was at $19.8^{\circ} \mathrm{C}$ and new-T2DM was at $18.5^{\circ} \mathrm{C}$. Figure represents the change of FPG at P10 (ref) of daily mean temperature compared with P50 and P50 (ref) of daily mean temperature compared with P90. Error bar represents $95 \% \mathrm{CI}$. 

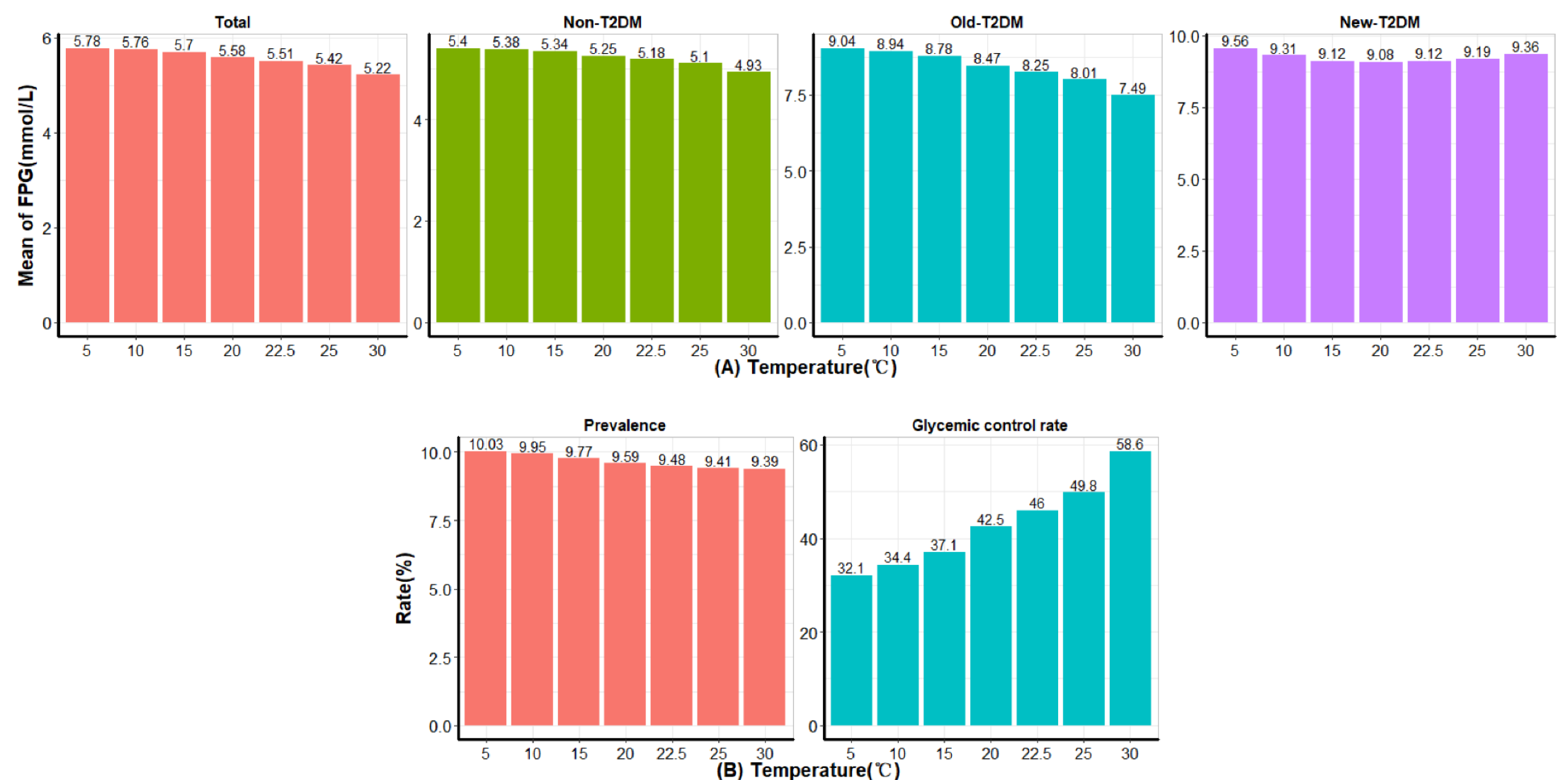

(B) Temperature $\left({ }^{\circ} \mathrm{C}\right)$

Figure 4. (A) Temperature adjusted FPG concentrations. (B) Temperature-FPG association adjusted prevalence (\%) and glycemic control rate (\%) of T2DM. Adjusted 


\section{Discussion}

289 In our study, we found that FPG levels were significantly higher in cold season than warm season among different populations, and the exposure-response curves between ambient temperature and FPG were downward parabola-shaped in total, non-T2DM and old-T2DM populations, while the curve was U-shaped in new T2DM population. The prevalence and glycemic control rate of T2DM varied across temperature. Temperature-FPG association adjusted T2DM prevalence decreased as temperature increased, while glycemic control rate of T2DM increased with the rising of temperature. This is the first large-scale study estimating temperature-FPG association adjusted prevalence and control rate of T2DM.

We found that FPG had apparent seasonal variation with high concentrations in cold season and low concentrations in warm season, which was consistent with previous studies $[5,6,15]$. For instance, a similar association was seen that the level of FPG was $0.6 \mathrm{mmol} / \mathrm{L}$ higher in winter $\left(13^{\circ} \mathrm{C}\right)$ than in summer $\left(23^{\circ} \mathrm{C}\right)$ in San Diego County,

302 California [5]. A large study included 15 middle and high countries also found that 303 higher FPG of adults was observed in winter than summer in both Northern Hemisphere and Southern Hemisphere [15].

305 Previous studies have reported that a negative association between ambient temperature and FPG [5, 7]. However, these researches just estimated their linear correlation coefficient rather than the actual curve linear relationship between ambient temperature and FPG, and they did not pay attention to the variation in sensitivity of temperature among various people, such as non-T2DM individuals and T2DM individuals. Our study therefore established their curve linear relationship and found that FPG concentration significantly decreased as temperature increased, while the rate of decent increased as temperature increased among total, non-T2DM and old T2DM populations. In addition, our results indicated that the influence of temperature on the FPG seems to be stronger in old T2DM populations than non-T2DM. The reason may be T2DM is associated with declining insulin sensitivity and beta-cell 316 function, and the glucose regulation of T2DM was more poorly than non-T2DM [16]. 317 For new T2DM population, we found that exposure-response curve of ambient temperature and FPG was U- shaped, but the effect of low and high temperature on FPG was not statistically significant. This result may be explained by that new T2DM 
patients were under large fluctuation in FPG without taking hypoglycemic medicine, which may affect the stability of FPG. Future prospective studies were needed to investigate this among new T2DM patients with and without medication treatment. Secondly, as the present study was conducted in winter, spring and autumn when there are limited days with high temperature, which may not find the extreme hot ambient temperature effect on FPG. Future studies can further observe the association of temperature and FPG in hot season or high temperature.

Related mechanism on the effects of ambient temperature is that temperature variation of two major hormones known to affect glucose homeostasis including plasma insulin and glucagon [17, 18]. Sustained exposure to low ambient temperature increase energy expenditure and insulin resistance $[19,20]$. Population study demonstrated that per $10^{\circ} \mathrm{C}$ increase of outdoor temperature was associated with 0.57 units increase of insulin sensitivity index [21]. Exposing extreme cold temperature could lead to increase in the thyrotropin and decrease in total thyroxine and free thyroxine (TF4), which related to decreased insulin sensitivity $[22,23]$.

Previous studies found that the incidence and glycemic control rate of T2DM varied with seasons, which may lead to underestimate of T2DM prevalence and overestimate of T2MD glycemic control rate when epidemiological surveys conducted in hot season $[6,9,10]$. To our knowledge, previous T2DM prevalence surveys rarely considered the effects of ambient temperature [11]. Our study estimated prevalence and glycemic control rate of T2DM after adjusting exposure response relationship of ambient temperature and FPG, and found that temperature-FPG association adjusted T2DM prevalence decline with the raise of temperature and glycemic control rate increase as temperature rose. These findings suggested the adjustment of temperature on FPG should be applied when estimating the prevalence and control rate of T2DM, as well as making nutritional prescriptions, exercise plans and treatment protocols for T2DM cases. For example, people with borderline high FPG in hot temperature would be diagnosed as T2DM in cold temperature, and the temperature and FPG association can help make the decision about starting early hypoglycemic treatment. The treatment for old T2DM patients may need to be adjusted in cold seasons.

Our study had several strengths. First, our study sample was relatively large. Secondly, 
351 we analyzed the associations between FPG and temperature among different 352 populations. Thirdly, we calculated the temperature-FPG association adjusted T2DM 353 prevalence and control rate. However, several limitations should be warranted in this 354 study. First, ambient temperature was the daily mean temperature at nearest 355 meteorological station which is not the real measurement of individual temperature 356 exposure. Second, our study was cross-sectional, which restricted our ability to 357 explore and precisely understand the causal influence of temperature on individual's 358 FPG. Thirdly, some confounding factors were not included in our study, such as 359 dietary structure and heredity. Fourth, we just have data from October to May, which 360 limited our ability to evaluate the associations between temperature and FPG at hot 361 seasons. 


\section{Conclusion}

363 In conclusion, FPG, prevalence and glycemic control rate of T2DM are affected by 364 ambient temperature, which suggests the ambient temperature-FPG association should 365 be considered and adjusted when estimating prevalence and glycemic control rate of 366 T2DM. For clinical implications of T2DM, the temperature and FPG association can 367 help improve the T2DM diagnosis and guide decisions regarding hypoglycemic 368 treatment to achieve normal FPG control. 
370 Additional file1: TABLE S1. Number (\%) of participants in each survey area in 371 Guangdong province during 2007 and 2016.

372 Additional file2: Figure S1. The non-linear relationships between daily mean, daily 373 minimum, daily maximum temperature and FPG among different populations.

374 Additional file3: Figure S2. The non-linear relationships between daily mean and 375 FPG among different population at $\mathrm{k}=3,4,5$.

376 Additional file4: Figure S3. The density distribution of fasting plasma glucose among 377 different population.

378 Additional file5: TABLE S2. Statistical description of fasting plasma glucose (FPG) 379 and daily mean ambient temperature among different populations.

380 Additional file6: TABLE S3. Number (\%), Mean and SE of fasting plasma glucose 381 and daily mean temperature according to month.

382 383

Additional file7: Figure S4. The exposure-response relationships between lag1 to lag6 of ambient temperature and FPG among different populations.

Additional file8: Figure S5. The exposure-response relationships between ambient temperature and FPG among different populations additionally adjusted sunshine and precipitation.

\section{Competing interests}

The authors have declared that no conflict of competing financial interests.

\section{Acknowledgements}

We would like to thank all the local CDC and community investigators and registered nurses for collecting baseline data and blood sample. We also thank all participants in this study.

\section{Authors contributions}

JL carried out the statistical analyses, JL and GH drafted the manuscript and contributed equally to this research. YX, ZC, XX, GJ, WM participated in study design and data collection. SC, JH, TL, WZ, XL, JX, LG, WM reviewed and revised manuscript. All authors approved final version of the manuscript to be published. 


\section{Funding}

399 This work was supported by the National Key Research and Development Program of 400 China [2018YFA0606200, 2018YFA0606202]; National Natural Science Foundation 401 of China [81874276, 81502819]; Natural Science Foundation of Guangdong, China 402 [2019A1515011880].

\section{Ethics approval}

404 The protocol was approved by the Ethics Committee of Guangdong Provincial Center 405 for Disease Control and Prevention (Ethical review code: 2019009). All the 406 participants agreed to participate and signed informed consents form prior to the 407 surveys.

\section{Author details}

$409{ }^{1}$ School of Public Health, Guangdong Pharmaceutical University, Guangzhou, China $410{ }^{2}$ Guangdong Provincial Institute of Public Health, Guangdong Provincial Center for 411 Disease Control and Prevention, Guangzhou, China

$412{ }^{3}$ Guangdong Provincial Center for Disease Control and Prevention, Guangzhou, China

$413{ }^{4}$ School of Public Health, Southern Medical University, Guangzhou, China

\section{Availability of data}

415 Data are not publicly available and information on how to access the data can contact 416 details: Email: mawj@gdiph.org.cn. 


\section{References}

418 1. International Diabetes Federation. IDF Diabetes Atlas, 8th edn. Brussels, Belgium: International Diabetes Federation, 2017. Available at: http://www.diabetesatlas.org.

10. Sakamoto M, Matsutani D, Minato S, Tsujimoto Y, Kayama Y, Takeda N, et al. Seasonal

12. Lao XQ, Xu YJ, Wong MC, Zhang YH, Ma WJ, Xu XJ, et al. Hypertension prevalence,

13. He Y, Wu W, Zheng HM, Li P, McDonald D, Sheng HF, et al. Regional variation limits

11. Wang L, Gao P, Zhang M, Huang Z, Zhang D, Deng Q, et al. Prevalence and Ethnic Pattern of Diabetes and Prediabetes in China in 2013. JAMA 2017;317(24):2515-2523. doi: 10.1001/jama.2017.7596. awareness, treatment, control and associated factors in a developing southern Chinese population: analysis of serial cross-sectional health survey data 2002-2010. Am J Hypertens 2013;26(11):1335-1345. doi: 10.1093/ajh/hpt111. applications of healthy gut microbiome reference ranges and disease models. Nat Med 2018;24(10):1532-1535. doi: 10.1038/s41591-018-0164-x.

14. World Health Organization. Definition and diagnosis of diabetes mellitus and intermediate 
hyperglycemia: Report of a WHO/IDF Consultation. WHO Document Production Services, Geneva, Switzerland, 2006.

15. Marti-Soler H, Gubelmann C, Aeschbacher S, Alves L, Bobak M, Bongard V, et al. Seasonality

16. Kahn SE. The relative contributions of insulin resistance and beta-cell dysfunction to the pathophysiology of Type 2 diabetes. Diabetologia 2003;46(1):3-19. doi: 10.1007/s00125-002-1009-0.

17. Campbell IT, Jarrett RJ, Rutland P, Stimmler LJD. The plasma insulin and growth hormone response to oral glucose: Diurnal and seasonal observations in the antarctic. Diabetologia 1975;11(2):147-150. doi: 10.1007/bf00429839.

18. Kuroshima A, Doi K, Ohno T. Seasonal variation of plasma glucagon concentrations in men. Jpn J Physiol 1979;29(6):661.doi: 10.2170/jjphysiol.29.661.

20. Cepeda M, Muka T, Ikram MA, Franco OH, Schoufour JD. Seasonality of Insulin Resistance,

21. Berglund L, Berne C, Svardsudd K, Svardsudd K, Garmo H, Melhus H, et al. Seasonal

22. Roos A, Bakker SJ, Links TP, Gans RO, Wolffenbuttel BH. Thyroid function is associated with between changes in serum thyrotropin and total and lipoprotein cholesterol with prolonged 